\title{
External representation of argumentation in CSCL and the management of cognitive load
}

\author{
J.M. van Bruggen *, P.A. Kirschner ${ }^{1}$, W. Jochems \\ Open University of the Netherlands, OTEC, P.O. Box 2960, 6401 DL Heerlen, Netherlands
}

\begin{abstract}
Computer-supported collaborative learning (CSCL) environments, particularly environments where students share external representations, are discussed as an interesting area for the application of cognitive load theory (CLT). CSCL environments share a number of characteristics that will induce considerable cognitive load. This article reflects on the characteristics of CSCL environments and external representations and the research opportunities they offer to CLT. We argue that CLT can contribute to our understanding of CSCL by pinpointing situations in which high levels of cognitive load are generated. CLT also raises a number of particular issues: from a CLT point of view studying of worked out external representations may lead to better learning outcomes than (co)-construction which is the standard mode of operation in CSCL. The ontology and specificity of the representational scheme used are crucial factors, as they define what can be expressed and how much room for interpretation is left in the representation. CLT may help identify mismatches between representational schemes and execution of (sub)tasks. CLT has lead to a number of recommendations on the integration of diagrammatic and textual information. We relate this to discussion and chat facilities in CSCL and describe how these can be more firmly anchored in the external representation. (C) 2001 Elsevier Science Ltd. All rights reserved.
\end{abstract}

Keywords: Cognitive load theory; Computer-Supported collaborative learning; External representations; Argumentation

\section{Introduction}

Cognitive load theory (CLT) (Sweller, van Merriënboer, \& Paas, 1998) has stimulated research on the presentation of information, in particular with respect to learn-

\footnotetext{
* Corresponding author.

E-mail address: jan.vanbruggen@ou.nl (J.M. van Bruggen).

1 Also at the Maastricht University.
} 
ing material. Within CLT, research on external representations has concentrated on pictorial representations embedded in the learning material: a separate presentation of text and its pictorial representation may lead to the so called split-attention effect. Integration of textual descriptions and pictorial representations will in general reduce the need to switch back and forth between the text and illustration, thus reducing or eliminating the split-attention effect.

Although a substantial amount of research in CLT is aimed at improving the learning material, the cognitive architecture assumed in CLT will not necessarily limit the application of the theory to the learning material alone. In this article we will concentrate on the applications of CLT in a particular area: shared external representations in computer-supported collaborative learning (CSCL) settings.

There are a number of reasons why CSCL environments using external representations are an interesting area for CLT. The primary reason is that CSCL environments force learners to coordinate one or more external representations. These representations may work as distinct sources of information that have to be integrated or at least coordinated in order to reach a common understanding. From a cognitive load perspective this may result in high-cognitive load, analogous to the split-attention effect that may occur when learners have to integrate the textual and pictorial information sources presented separately. Asynchronous discussions using basic discussion group functionality would seem vulnerable to this effect, because the reader has to maintain a coherent and consistent trace of the discussion by referring back and forth to the previous entries in the discussion and possibly to the external material. Establishing and maintaining a common ground is at the center of collaborative learning (Baker, Hansen, Joiner, \& Traum, 1999). Grounding and collaborative use of external representations have associated additional costs: external representations that are produced for someone else, tend to be more complete and richer in annotation than representations produced for personal use (Cox, 1999).

The very nature of collaborative learning (or working) thus seems to induce cognitive load, on an individual level, as well as shared. Within CSCL environments, where students share external representations, some measures can be taken to reduce extraneous cognitive load: the features of external representations may reduce demands on cognitive capacity, sharing external representations and interaction management may lead to increased task focus.

This article tries to identify a number of characteristics of CSCL environments and external representations used in those environments. We will then show how these characteristics may influence cognitive load and the measures that could be taken to maintain desirable levels of cognitive load.

\section{Cognitive load theory}

CLT assumes a limited working memory connected to an unlimited long-term memory (Baddeley, 1986). Working memory embodies at least two mode-specific components: the visuospatial sketchpad; and the phonological loop. Further, it incorporates a coordinative component: the central executive. Knowledge is organized 
and stored in long-term memory in the form of schemata. A schema can hold a huge amount of information, yet is processed as a single unit in working memory. Schemata can integrate information elements and production rules and become automated, thus requiring less storage and controlled processing. Basically, CLT is concerned with the limitations of working-memory capacity and the measures that can be taken to promote learning, that is the construction of schemata, by imposing adequate levels of cognitive load.

The factors that determine the level of cognitive load are described by Paas and van Merriënboer (1994) (see Fig. 1). They distinguish causal and assessment factors. Causal factors include characteristics of the subject (e.g. cognitive abilities), the task (e.g. task complexity), the environment (e.g. noise), and their mutual relations. With respect to assessment factors, Paas and van Merriënboer (1994) distinguish mental load, mental effort, and performance as the three measurable dimensions of cognitive load. Mental load is the portion of cognitive load that is imposed exclusively by the task and environmental demands. Mental effort refers to the cognitive capacity actually allocated to the task. The subject's performance, finally, is a reflection of mental load, mental effort, and the aforementioned causal factors.

Learning, which is reflected by performance change, requires working-memory capacity. That is, it imposes a germane cognitive load on the learner (Sweller et al., 1998). Germane cognitive load is required for an essential aspect of learning, namely the construction and storage of schemata into long-term memory. The construction of adequate and rich schemata is especially important in complex learning tasks where it will require more effort, because the elements contained by the to-be-learned material are highly interconnected. This is referred to as intrinsic cognitive load, which is the portion of load that is imposed by the intrinsic characteristics of the task or subject matter. According to CLT the limitations of working memory are rarely taken into account in conventional instruction. Conventional instructions tend

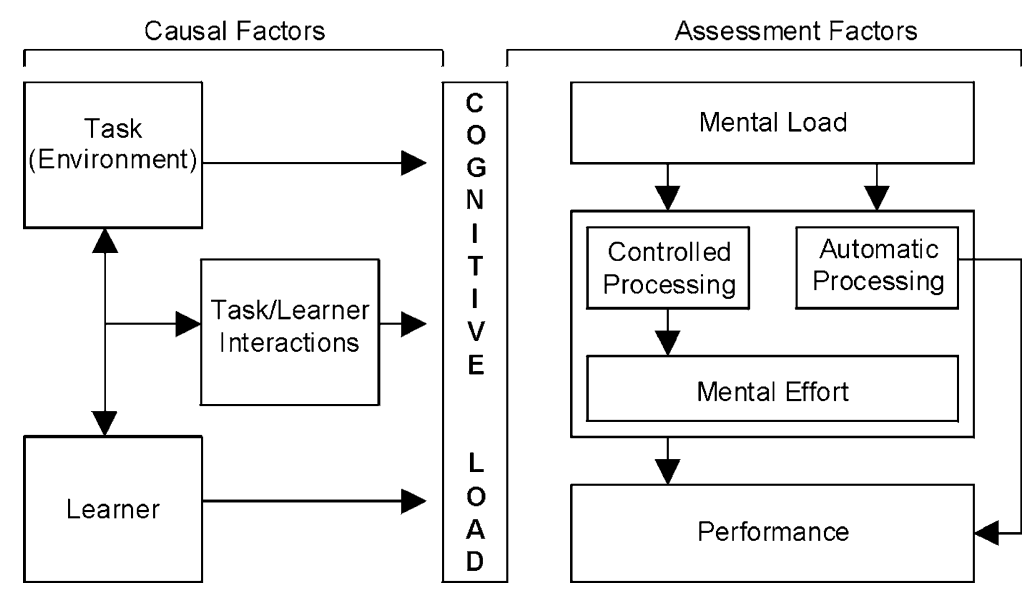

Fig. 1. Causal and assessment factors of cognitive load (Adapted from Paas \& van Merriënboer, 1994). 
to impose an extraneous cognitive load on working memory, whereas learning something requires shifting from extraneous to germane cognitive load.

CLT has lead to the development of a number of instructional formats, including goal-free problems, worked examples and completion problems. An exhaustive overview of CLT-based instructional formats and their empirical base is given by Sweller et al. (1998). We single out one caveat, that seems most relevant in the context of CSCL: avoid split-attention. Instructions often comprise mutually referring sources of information, which force learners to split their attention (between a diagram and a textual description, for instance). If neither of these two information sources can be understood on their own, they have to be mentally integrated. This integration process imposes a high extraneous load. Physically merging the sources will reduce this unnecessary mental load.

We will argue that within the CSCL environments it is important to consider whether the cognitive load imposed on learners is such that it fosters learning.

\section{Characteristics of computer-supported collaborative learning}

CSCL is defined in various ways, ranging from computer supported joint efforts to solve problems, to processes that help students become members of knowledge communities (Koschmann, 1996). Despite large differences of opinion on the final aim of CSCL, there are a number of common elements found in many CSCL environments. In general, it involves groups of students distributed across different locations working together on a common problem or project. This process is supported by specific software and/or environments, that run the gamut from simple asynchronous discussion lists, through groupware for asynchronous work to synchronous multimedia environments with sharing of workspaces, programs and documents.

Kolodner and Guzdial (1996) argue that three characteristics are typical to CSCL software, namely the interface, the representations and the function that the software tries to achieve. CSCL interfaces are interfaces for learners and require emphasizing structure for novices who need guidance to succeed and flexibility for students with diverse needs. CSCL needs (multiple) representations that lend themselves to extrapolation and discussion. CSCL software can support different functions: synchronous or asynchronous collaborations; and local or remote collaboration. Kolodner and Guzdial also note that CSCL can fulfil a number of roles, namely (a) promoting inquiry and sense-making; (b) facilitating knowledge building by providing a forum for collaboratively presenting arguments, raising learning issues, and reaching consensus on new knowledge; (c) record-keeping and/or external memory functions; (d) enabling communication with distant communities; (e) promoting reflection of alternative perspectives, solutions, and critiques, and (f) supporting teacher planning and implementation of collaborative activities.

CLT as an analysis tool for CSCL is not an obvious choice given these roles (or the higher level goals whose attainment CSCL tries to facilitate) and the different theoretical backgrounds. CSCL is based on constructivist theories, whereas CLT is rooted in cognitive theory. The two meet, we think, in the use of external represen- 
tations. External representations are promoted in CSCL as 'augmenting' cognitive activity (Pea, 1993) and as offering new views, for instance by visualization, (Jonassen, Peck, \& Wilson, 1999). As pointed out by Duffy and Cunningham (1996) one impact of this view is that 'off-loading' basic cognitive demands may permit the learner to attend to higher-level representations. CLT can help us understand why offering external representations by itself does not necessarily offer this 'offloading' effect.

In this article we will concentrate on the representation of argumentation in CSCL, particularly in the ill-structured domains of scientific inquiry and design, i.e. the roles (a), (b) and (e) referred to above and the use of multiple representations in CSCL and consider the 'off-loading' effects.

We will give a brief overview of cognitive factors of external representations and characteristics of external representations. We then describe the various uses of external representations of argumentation in CSCL systems and conclude with a number of tentative statements regarding the use of CLT in CSCL.

\section{Cognitive factors of external representations}

Zhang (1997, p. 180) defines external representations very broadly as "the knowledge and structure in the environments, as physical symbols, objects, or dimensions (e.g. written symbols, beads of abacus, and dimensions of a graph) and as external rules, constraints, or relations embedded in physical configurations (e.g. spatial relations of written digits, visual and spatial layouts of diagrams, and physical constraints in abacuses)". Larkin and Simon (1995) characterize the information preserved in diagrammatic and sentential representations of a problem: diagrammatic representations preserve information about the topological and geometrical relations between components of the problem, whereas sentential representations represent a natural language representation of the problem, which may contain information on temporal or logical sequence. On this basis, graphical representations are described as to "include diagrams, maps, plans, animations and virtual reality and... [to be] distinct from propositional/sentential representations and formal notations" (Scaife \& Rogers, 1996, p. 186). The type of representations that we are going to discuss in this article are of a semi-formal nature: they tend to use a restricted set of objects and relations, but rely on natural language specification of their meanings.

There have been various claims with respect to the cognitive basis of the effectiveness of external, graphical representations. Larkin and Simon (1995) showed that diagrammatic representations require less search, comprehension and inference than sentential representations. Cox (1999) in his review of external representations, discusses studies indicating that graphical representations make use of a visuospatial scratchpad, which may reduce the working memory load. These claims are based on a single agent model of internal representation. Zhang and Norman (1994) emphasize the distributed character of representations, and the effects that external representations can have on task performance (e.g. using Roman numerals instead of Arab numerals in multiplication). In their study of Tower of Hanoi isomorphs, 
Zhang and Norman claim that external representations provide the following: (a) memory aids; (b) information that can be directly perceived and used without being interpreted and formulated explicitly — the rules are built into the representations rather than formulated; (c) anchor and structure cognitive behavior, i.e. representations with built-in rules constrain possible actions; and (d) change the task, because one has to process less rules. Zhang (1997) claims that perceptual saliency characteristics of external representations can lead to biases in processing, though not necessarily in favor of a solution. Zhang and Norman's work has influenced the development of Belvédère, an environment for collaborative scientific inquiry, where current work is directed at trying to exploit 'perceptual salience' by offering alternative representations to the 'evidence map' (Suthers, 1998a).

Scaife and Rogers (1996) point out that these and other claims on the effectiveness of external graphical representations rest on different assumptions about cognitive architecture. They question empirical and theoretical evidence behind much of the research on external representation. Further, according to Scaife and Rogers we still lack an adequate theoretical account of the processes involved in the interaction with external representations.

\section{Characteristics of representational schemes}

The term representational scheme is used here to refer to the semi-formal notations used to construct external representations in the CSCL-environments we are considering. We use the term representation scheme rather than formalism, as the representations offered in the environments to be discussed seem to be more on a conceptual level and are less formal than for instance the Euler circles or models offered in other environments. A number of general characteristics or dimensions of external representations apply here: perspective (and closely related ontology), precision, modality - the form of expression used for displaying information sucu as text, animations, and graphs — specificity, and complexity (de Jong et al., 1998). In this discussion we will focus on the ontology, specificity and precision aspects of the way in which argumentation can be represented.

The ontology of a representation defines what can be seen in the domain represented and how it will be seen. The ontology "refers to the content, to the objects and relations one uses to represent a domain, not so much to the symbols by which objects and relations are denoted" (de Jong et al., 1998, p. 11).

Specificity is defined by Stenning and Oberlander (1995, p. 98) as "the demand of a system of representation that information in some class be specified in any interpretable representation". The specificity of a representation may require disambiguation: we cannot represent in a diagram that an object is 'next to' another object, it has to be represented at a particular locality. Thus, graphical representations limit abstraction and aid processibility of the information. Cox formulates this as a design guideline: "select a representation with sufficient expressive power to capture the indeterminacy in the problem, but no more than is needed" (Cox, 1999, p. 350).

The precision of a representation reflects how accurately the representation reflects 
the underlying model. In science and mathematics domains this is related to quantitative vs. qualitative models. In other domains it will reflect the nature of the objects, e.g. differentiating between a hypothesis and a prediction and their relations, e.g. where various relation types are made available. We will provide examples of the ontology and specificity of external representations used in CSCL environments and how they can assist the learning process, or interfere with it.

\section{Coordinating representations}

In CSCL using external representations students are faced with the need to somehow integrate or at least coordinate several representations. Stenning (1998) makes a relevant distinction between surface representations and conceptual representations. Differences between surface representations reflect different types of representation, e.g. by Venn diagrams or Euler circles, of the same underlying conceptual system. Differences between conceptual representations refer to differences in conceptual systems, for instance different theories about the nature of light. These differences may become more clear when the conceptual systems are represented using the same surface, like the evidence maps of Belvédère or the claim frames of SenseMaker.

When we discuss coordinating representations, we can differentiate between a number of situations. In the first place, students may have to coordinate multiple surface representations of the same underlying conceptual domain. This is not a trivial task: students fail to integrate textual and graphical representations of economic laws (Schijf \& Simon, 1998). In the second place students may fail to integrate different conceptual representations, operating on 'islands of knowledge'. Medical students have great difficulty in integrating different bodies of knowledge (e.g. biomedical, clinical) in diagnosis (Boshuizen \& van der Wiel, 1998). If a task is very complex, students tend to rely on procedural aspects of task completion. Analysis of the use students make of the coaching component of the Sherlock troubleshooting system shows that only in the reflection phase — a sort of 'post-mortem' session students use the 'WHY'-type of coaching Sherlock offers (Lesgold, 1998; Lesgold, Katz, Greenberg, Hughes, and Eggan, 1991), i.e. address coordination with a deeper domain knowledge.

New situations arise in collaborative settings, where multiple agents have to coordinate multiple representations, both conceptual and surface. They may not have to completely integrate their individual representations, but at least they have to establish a partially shared representation. There is reason to assume that coordination, and certainly integration, has associated cognitive costs (Boshuizen \& Schijf, 1998).

One way to support coordination in CSCL environments is adding structured dialogue interfaces. Replacing the chat box in the C-CHENE system with a structured dialogue interface led to more reflective interactions between students (Baker \& Lund, 1997). We will give more examples of the way in which CSCL environments try to facilitate the coordination process. 


\subsection{Representation of argumentation in computer-supported collaboration}

We now turn to the representation that is at the core of our interest: representation of argumentation. Argumentation is represented in various ways in CSCL environments. Bell (1997) makes a distinction between discussion-based tools and knowledge representation tools. Discussion-based tools, e.g. CSILE (Scardamalia \& Bereiter, 1994; Scardamalia, Bereiter, Maclean, Swallow, \& Woodruff, 1989) and the Collaboratory Notebook (Edelson \& O’Neill, 1994; Edelson, O’Neill, Gomez, and D’Amico, 1995) support dialogical argumentation of a group. Knowledge representation tools, such as SenseMaker (Bell, 1997) and Belvédère (Paolucci, Suthers, \& Weiner, 1995; Suthers \& Weiner, 1995) have to support the construction of rhetorical argumentation by individuals as well. Further, the tools have to support argumentation whose structure and content correspond to, in this case, a valid scientific argumentation.

\subsection{Representation in knowledge representation tools}

The representations used in the knowledge representation tools, can be described with the characteristics of external representations presented above (ontology, specificity, precision). We will illustrate this with the representations of argumentation found in the Belvédère system (Paolucci et al., 1995). Belvédère is a system to support collaborative scientific inquiry. Learners are confronted with challenge problems (e.g. What caused the extinction of the Dinosaurs?) that need a scientific explanation. The Belvédère environment offers access to web-based material, a chat window, and a shared visual workspace where learners construct scientific explanations in so-called 'evidence maps' (Suthers, Toth, \& Weiner, 1997). The characteristics of the notation of the evidence maps is what we concentrate on here.

The ontology of the evidence maps in Belvédère is inspired by a Toulminian perspective on argumentation, with claims, warrants, backings, et cetera (Toulmin, 1958; Toulmin, Rieke, \& Janik, 1984). When students create evidence maps they can use a number of objects (principle, hypothesis, data, unspecified) and a number of simplified relations (for, against, and). Furthermore they can express how strong their beliefs in the objects and relations are. This obviously limits the scope of what can be expressed in Belvédère. Suthers (1998b) mentions several possible extensions to the representations in Belvédère like concept mapping, plan diagrams, causal loop diagrams, mathematical models, as well as 'language arts' with which students can construct diagrams according to their writing goals. Some of these extensions add support to the representation of the domain (concept mapping, mathematical models, causal loop), i.e. widen the scope of the ontology, while others are added to support student activities within these domains.

The evolution of Belvédère offers examples of the specificity and precision of the representational scheme as well. Suthers (1995) notes that learners are forced by the representational system used in Belvédère to indicate which type of object they add (the specificity of the representation forces disambiguation). This often leads to epistemological discussions between learners (that will not be represented in the evidence 
maps however). Suthers points out that a weaker representational structure could evade the issue, but that this would also obscure the need to discuss these important points. In terms of specificity, a weaker representation would leave room for different (implicit) interpretations of the representation. In subsequent versions of Belvédère the number of objects and especially the different types of relations (precision of the representation) were reduced, partly because they were considered redundant, partly because the detailed level at which relations could be presented seemed to interfere with the task - caused students to deal with non-goal tasks (Suthers et al., 1997). There is some evidence from other areas in which collaborative argumentation is used that the representation scheme employed may interfere with task performance. In their review of argumentation-based design rationale methods Buckingham Shum and Hammond (1993) mention studies where issue-based notations sidetracked designers from construction, or where QOC (Questions, Options, Criteria) with its emphasis on stating design alternatives interfered with top-down refinement of design.

The Belvédère examples show that a cognitive 'off-loading' effect is not something that comes naturally with an external representation. To the contrary: characteristics of the external representation seem to impose extraneous cognitive load and may lead to activities that do not foster any deeper representation of the domain. In this example the characteristics of the external representation seem to have added to the coordination problem in the CSCL environment. Recent research using the Belvédère environment by Veerman (2000) and Veerman and Treasure-Jones (1999) showed how complex the relations are between the 'chat'-exchanges (the coordination platform) and the contents of the shared evidence maps.

\subsection{Representation in discussion-based tools}

Discussion-based tools offer the students an environment in which they can exchange arguments. The structure of the argumentation is not explicitly represented, i.e. it may be embedded in the threads of an electronic discussion. One of the best known discussion-based environment is CSILE (Scardamalia \& Bereiter, 1994; Scardamalia et al., 1989). In CSILE students use a communal database of interrelated text and graphic notes. When working with a recognized problem, students are required to enter notes with identified type of content: "My Theory", "I Need To Understand", "New Information", "Comment". All notes are entered in the communal database and are available to other students for search and comment. CSILE can offer a number of views (diagrams, maps) to which a network of related notes can be attached. The Collaboratory Notebook (Edelson \& O’Neill, 1994; Edelson et al., 1995) is a shared workspace in which learners can enter pages and relate them through hyperlinks. There are several types of pages, including: "question", "conjecture", "evidence for", "evidence against". The discussion in the Collaboratory Notebook is scaffolded by an interface that suggests particular follow-up pages (e.g. an 'evidence for' page as a follow-up to a conjecture page). Discussions threads in asynchronous discussions tend to be short (Guzdial, 1997), but they can be lengthened by anchoring discussion 
in relevant material, as is being done in the CaMILE environment (Guzdial et al., 1997).

An approach that is somewhere between representation tools and discussion-based tools is taken by Baker and Lund (1997). They offered learners a structured communication interface to the C-CHENE systems for collaborative solving of energy flow problems. In the structured communication interface the learner characterizes the type of interaction s/he is pursuing (construct, propose, manage the interaction). Baker and Lund found that students could use this type of interface, and - although the quality of solutions showed no difference between groups - that students working with the structured interface produced more task focussed and reflective interactions (explanation, justification, evaluation). We refer the reader to Veerman and Treasure-Jones (1999) for a review of task and system characteristics related to provoking and supporting argumentation.

In discussion-based environments visual traces of the discussion lines and signaling the different argumentation moves by node types are means to achieve cognitive off-loading. They can assist students in maintaining a common focus on the argumentation. The structured dialogue interfaces have a similar function.

\section{External representations to support argumentation in design}

Most of the CSCL environments referred to above were aiming to support scientific inquiry. Although the domain of scientific inquiry is ill-structured, at least the objects and the structure of the resulting argumentation show considerable overlap between the systems. A scientific explanation requires theories, hypotheses and data to be represented, as well as the support/oppose relations that may exist between them. In design, alternatives have to be evaluated against criteria and decisions have to be made to select those alternatives that will reach the goals, within the constraints the designer has to operate in. Design problems often are ill-structured problems, characterized by high levels of uncertainty about the actual goals, criteria and stoprules that can be applied (Goel \& Pirolli, 1992; Rittel \& Webber, 1984). Design rationale is a systematic approach to lay out the reasons and the reasoning behind the decisions that lead to the design of an artifact (Buckingham Shum \& Hammond, 1993; Caroll \& Moran, 1991). In order to represent the topics under consideration, as well as the argumentation for or against their (partial) solutions, a number of representational schemes were developed to graphically represent argumentation (Buckingham Shum, MacLean, Bellotti, \& Hammond, 1997).

Issue based information system (IBIS), is a design approach developed by Rittel (1984) which engages the stakeholders in discussing problems, resolving problems, elucidating concerns and posing questions. A graphical representation of Issues, Positions (pro/con) and Arguments is at the core of g(raphical)IBIS, and later the commercially available QuestMap system (Conklin \& Begeman, 1988; Conklin \& Yakemovic, 1991). Specializing the IBIS-type notation to serve software design QOC (Questions, Options, Criteria) notation was developed for use in Design space analysis (DSA). DSA aims to have the alternatives (options) for a design carefully enumer- 
ated and evaluated on the basis of a set of explicitly defined criteria, thus laying out the 'design space'. Coherent design 'spaces' are defined for future re-use. QOC stands for Questions (and consequent questions), the Options (alternatives) and the criteria by which options can be evaluated (Buckingham Shum et al., 1997; MacLean, Young, Bellotti, \& Moran, 1991a,b).

A more generic approach to representing design argumentation is proposed by Lee and Lai (1991). Their decision representation language (DRL) was developed to support decision making, but can be shown to represent several 'spaces' of design rationale in argument, alternative, evaluation, criteria and issue spaces. Lee and Lai (1991) give detailed examples of the design rationale questions that DRL supports, using the 'spaces' in the design rationale representation. The core objects in DRL are decision problem, alternative, (sub)goal (which represents criteria), question, and claims. All relations in DRL are specializations of the claim object: achieve(alternative, goal), supports(claim, claim), denies(claim, claim), presupposes(claim, claim).

DRL could (and Lee and Lai state it should) be specialized for use in particular domains. Let us consider what that could mean by taking, again, Belvédère as an example. Assume someone has drawn a relation between a hypothesis and data to support it. Now we want to undercut this support, because we question the reliability of the measurement. In Belvédère we would then draw an 'against'-line from an object (say a datum that the reliability of the measurement was low) to the relation between data and hypothesis. In DRL the support-relation itself is a claim object to which we can relate any further discussion, for instance that it presupposes reliable measurement. We can as well lay out a series of goals (criteria) that we are using to decide between the best explanation: parsimony, reliability and validity, et cetera and discuss whether the claims in the argumentation help reaching these goals. DRL thus seems flexible enough to deal with different types of domains, and for that reasons we have chosen to explore its use in further research.

Two final notes to this section: (a) The representation of the argumentation in all schemes discussed does not support modeling the argumentation in an ongoing debate. This will require the advanced modeling found in systems such as EUCLID (Smolensky, Fox, King, \& Lewis, 1988). (b) The notational schemes used for design argumentation are sufficiently weak to support argumentation in other domains, under a general heading of computer-supported collaborative argumentation - a field of study that is now becoming integrated in CSCL (Carr, 1999; Zumbach \& Reimann, 1999).

\section{External representations and cognitive load}

We will now turn to the use of external representations and its implications from a cognitive load perspective. The basic questions here are whether the use of an external representation scheme may lead to a change in extraneous cognitive load and whether it will lead to a change in germane cognitive load. Consider the use of an external representation by a person who is using this scheme individually. Such 
a scheme may act as an external memory, and subjectively, may function properly, even though from an outsider's view the representation does not look adequate. Now turn to a collaborative setting in which the representation will have to be shared by another person. As long as the symbol system and what it expresses are well known to the participants, say a simple calculation, sharing the representation may be relatively easy, although one might spend more time on making the representation readable and understandable to others (Cox, 1999). Sharing a representation thus brings additional (cognitive) costs. The question is whether there are profits as well. We are not pretending that we can give an answer to this question, we will point to a number of issues and questions that can be addressed by CLT research.

\subsection{Construction of external representations}

The benefits of active (co)-construction of external representations are more or less taken for granted in the CSCL community. The costs of preparing and coordinating representations that are associated with (co)-construction may however be considerable. In CSCL environments, especially in representation-based tools, the number of information elements may impose a considerable load: (a) learners have to coordinate multiple representations - in Belvédère, for instance, learners have to coordinate a research question with descriptions of relevant research made available in web pages; (b) learners have to extract the information (hypothesis, data) in the descriptions; and (c) map the extracted information onto the elements of an external representation (element type, relations with elements already in the evidence map), while d) adhering to the syntax and semantics of the external representation.

According to CLT the cognitive load in complex tasks, where the learner has to maintain several information items in working memory, may become so high that it will prevent the formation of schemata. In CLT the positive effect of goal-free problems, worked examples and completion problems is attributed to the prevention of a means-ends approach to problem solving which will eliminate the need to maintain a set of intermediate problem states in working memory. Could, therefore, within a CSCL context joint studying and discussion of worked examples replace (co)construction - at least in introductory phases? Or, would mapping back from the external representation to the background material be such a complex task that we trade in one type of extraneous cognitive load for another? Or, should we differentiate between the ontologies of representational schemes used, e.g. would schemes that are soliciting alternative views (IBIS, QOC) show better outcomes in (co)-construction conditions?

\subsection{Synchronous vs. asynchronous communication}

One of the first things to consider in CSCL environments are the tools (software) and communication modes (synchronous, asynchronous) available to the learners to create and exchange representations, and coordinate their interactions. CLT suggests that synchronous communication modes may induce high cognitive load because the load is imposed by the information as well as the pace at which it is presented by 
communicating partners. The latter factor may play a role in asynchronous communication as well: a rapid flow of notes and messages between students will induce high levels of cognitive load. In the design rationale literature coming to terms with the 'cognitive overhead' of real-time capturing of the design rationale is seen as one of the major challenges of implementing design rationale (Buckingham Shum \& Hammond, 1993; Buckingham Shum et al., 1997). Multi-modal synchronous communication in CSCL may offers means by which the modality effect can be exploited. As far as we know, research has yet to start tackling these issues.

\subsection{Characteristics of the representational scheme}

The characteristics of the representational scheme itself may induce cognitive costs. Buckingham Shum had software designers formulate QOC whilst working on software design. In those phases of the design where main issues were involved, QOC was seen by the designers as a valuable tool to help explicate goals, and support decision making. In more constraint tasks, e.g. top-down refining a design, formulating QOC seemed to interfere with the design task. Buckingham Shum interprets this finding in terms of the type of problem the designers were trying to solve: answering the major questions meant solving 'wicked problems', i.e. the type of problems IBIS and QOC were developed for. Refining the design was, according to Buckingham Shum, primarily a problem of checking design against already clearly defined constraints and making further refinements, which is outside the view defined by the ontology of the QOC representation (Buckingham Shum \& Hammond, 1993).

Similar experiences were gained with the first versions of the representational scheme embedded in Belvédère: the specificity of the representational scheme more or less forced learners to reflect on the type of object they were entering (principle, hypothesis) in their evidence map, which was considered a worthwhile goal. The representational scheme offered various types of relations and this seemed to sidetrack learners to off-task activities like discussing the details of the different types of relations available in the representational scheme.

What these observations seem to suggest is that the ontology and specificity of a representational scheme should be carefully matched not only to the desired outcome, but to the current state of task execution as well. This is a refinement of Cox' guideline to select a minimal representational scheme "with sufficient expressive power to capture the indeterminacy in the problem, but no more than is needed" (Cox, 1999, p. 350). This does not necessarily imply that different representational schemes have to be used. One can imagine that an environment scaffolds the process by zooming in on those parts of the representation that are relevant to the current state, for instance one of the spaces in a DRL representation of design rationale.

Belvédère's structure and content coaches provide us with other examples (Paolucci et al., 1995; Paolucci, Suthers, Weiner, \& Lesgold, 1996). The structure coach identifies patterns in the current representation, e.g. 'confirmation bias' if only data in favor of a particular hypothesis is entered into the evidence map. The coach then draws attention to the parts of the representation involved, presents a 'diagnosis' and offers suggestion on steps the learner could take to improve the evidence map. 
The content coach compares the current evidence maps with expert solutions, and can hint to evidence that the learners have not included, or to objects in the evidence maps they have linked in other ways that the experts have (Toth, Suthers, \& Weiner, 1997). By drawing attention only to the relevant aspects of the representation, and pointing out difficulties with the current evidence map, the coaches help maintain focus.

\subsection{Anchoring and structuring discussion}

As we mentioned before, synchronous communication modes are likely to add to the cognitive load of a task, if only because subjects have to adapt to a temporal sequence and a pace set by someone else. Discussion-based environments, using asynchronous communication modes can reduce this load, simply by offering the learner more time.

They do however create other costs for the learner. Students still face the problem of maintaining coherence and consistency in the discussion. Structured histories of the notes exchanged and maintaining different viewpoints on related notes may help reduce these costs. One common way to improve to the coherence and consistency of the discussion is by limiting the choice of a response type, a strategy found in CSILE and the Collaboratory Notebook. Another strategy is to allow a note to be connected to multiple notes. Viewpoints on the representation and, in a discussionbased environment like CSILE, 'rising above' notes may reduce the search space for the learner and help chunking information. Anchoring discussions in relevant material and preferably in an integrated interface, e.g. CaMILE (Guzdial, 1997) has been shown to increase the length of discussion threads.

In terms of CLT, the environments force the learner to search for elements that need to be mentally integrated, and CLT is very specific in its remedy for this 'splitattention effect': it recommends physically integrating information elements that have to be processed together. This may lead to a number of interesting interfaces for CSCL. Consider the interface for anchored discussion used in the on-line Journal of Interactive Media in Education (JIME — http://www-jime.open.ac.uk/) where the text outline, the text of the article, and the review discussion (threads) are presented in three window panes. For representation tools CLT seems to suggest, but this is also a question open to research, that better results can be expected if separate chat boxes and listings of chat history are replaced by 'chat threads' that are directly, visually related to the external representation (cf. the structured communication interface for C-CHENE (Baker \& Lund, 1997) which takes steps in this direction). Again, these are issues that are barely covered in the research literature.

\section{Conclusion}

In this article we reflect on the relations between the use of external representations in CSCL environments and CLT, and we admit that there is little empirical evidence collected in the area on which to base our reflections. There are good reasons for 
empirical research. Consider some of the roles CSCL software has to accomplish, according to Kolodner and Guzdial (1996): promoting inquiry and sense-making; facilitating knowledge building, and promoting reflection of alternative perspectives, solutions, and critiques. Given these roles, it comes as no surprise that learners in CSCL are confronted with complex, demanding tasks, often in complex environments.

CLT tells us that in these situations with high element interactivity reducing extraneous cognitive load may become essential in order for learning to occur. We have pointed to a number of areas in which CLT can contribute to learning in CSCL environments. In general, it can help pinpoint situations where the environments are making heavy demands on cognitive resources. It also leads to a number of more specific questions for research: (a) Would (joint) studying worked examples and completion problems of external representations in CSCL environment show the same positive effects on learning outcomes as had been found with instructional material, and what is the role of the ontology and specificity of the representational scheme used? (b) Can the modality effect be exploited in synchronous CSCL environments, and under what conditions? (c) Can we measure cognitive load induced by the ontology and specificity of representational schemes, in particular where the specificity is insufficient, i.e. leaving open multiple interpretations? (d) Can we apply CLT to create more firmly anchored and integrated discussions in CSCL environments and do they lead to better learning outcomes?

Addressing these questions will not only improve our understanding of CSCL environments: it may also help to further develop CLT in areas of distributed cognition and a wider range of external representations.

\section{References}

Baddeley, A. D. (1986). Working memory. Oxford: Clarendon Press.

Baker, M., Hansen, T., Joiner, R., \& Traum, D. (1999). The role of grounding in collaborative learning tasks. In P. Dillenbourg (Ed.), Collaborative learning: cognitive and computational approaches (pp. 31-63). Amsterdam: Pergamon Press.

Baker, M., \& Lund, K. (1997). Promoting reflective interactions in a CSCL environment. Journal of Computer Assisted Learning, 13, 175-193.

Bell, P. (1997). Using argument representations to make thinking visible for individuals and groups. In R. Hall, N. Miyake, \& N. Enyedy (Eds.), Proceedings of CSCL '97: The Second International Conference on Computer Support for Collaborative Learning (pp. 10-19). Toronto: University of Toronto Press.

Boshuizen, H. P. A., \& Schijf, H. J. M. (1998). Problem solving with multiple representations by multiple and single agents: an analysis of the issues involved. In M. W. van Someren, P. Reimann, H. P. A Boshuizen, \& T. de Jong (Eds.), Learning with multiple representations (pp. 137-151). Amsterdam: Pergamon Press.

Boshuizen, H. P. A., \& van der Wiel, W. J. (1998). Using multiple representations in medicine: how students struggle with them. In M. W. van Someren, P. Reimann, H. P. A. Boshuizen, \& T. de Jong (Eds.), Learning with multiple representations (pp. 237-262). Amsterdam: Pergamon Press.

Buckingham Shum, S., \& Hammond, N. (1993). Argumentation-based design rationale: what use at what cost? International journal of man-machine studies, 40, 603-652.

Buckingham Shum, S. J., MacLean, A., Bellotti, V. M. E., \& Hammond, N. V. (1997). Graphical argumentation and design cognition. Human-Computer Interaction, 12, 267-300. 
Caroll, J. M., \& Moran, T. P. (1991). Introduction to this special issue on design rationale. HumanComputer Interaction, 6, 197-200.

Carr, C. S. (1999). CSCA in legal education. In Computer Supported Collaborative Learning '99, Stanford, CA.

Conklin, E. J., \& Begeman, M. L. (1988). gIBIS: a hypertext tool for the exploratory policy discussion. ACM Transactions on Office Information Systems, 6, 303-331.

Conklin, E. J., \& Yakemovic, K. B. (1991). A process-oriented approach to design rationale. HumanComputer Interaction, 6, 357-391.

Cox, R. (1999). Representation construction, externalised cognition and individual differences. Learning and Instruction, 9, 343-363.

de Jong, T., Ainsworth, S., Dobson, M., van der Hulst, A., Levonen, J., Reimann, P., Sime, J.-A., van Someren, M. W., Spada, H., \& Swaak, J. (1998). Acquiring knowledge in science and mathematics: the use of multiple representations in technology-based learning environments. In M. W. van Someren, P. Reimann, H. P. A. Boshuizen, \& T. de Jong (Eds.), Learning with multiple representations (pp. 9-40). Amsterdam: Pergamon Press.

Duffy, T. M., \& Cunningham, D. J. (1996). Constructivism: implications for the design and delivery of instruction. In D. H. Jonassen (Ed.), Handbook of research for educational communications and technology (pp. 170-198). New York: Macmillan Library Reference USA.

Edelson, D., \& O'Neill, D. K. (1994). The CoVis collaboratory notebook: computer support for scientific inquiry. Paper presented at the Annual Meeting of the American Educational Research Association, New Orleans, April 1994. New Orleans.

Edelson, D. C., O’Neill, D. K., Gomez, L. M., \& D’Amico, L. (1995). A design for effective support of inquiry and collaboration. In CSCL 95, Bloomington, IN.

Goel, V., \& Pirolli, P. (1992). The structure of design problem spaces. Cognitive Science, 16, 395-429.

Guzdial, M. (1997). Information ecology of collaborations in educational settings: influence of tool. In R. Hall, N. Miyake, \& N. Enyedy (Eds.), Proceedings of CSCL '97: The Second International Conference on Computer Support for Collaborative Learning (pp. 83-91). Toronto: University of Toronto Press.

Guzdial, M., Hmelo, C., Hübscher, R., Nagel, K., Newstetter, W., Puntambekar, S., Shabo, A., Turns, J., \& Kolodner, J. (1997). Integrating and guiding collaboration: lessons learned in computer-supported collaborative learning research at Georgia Tech. In R. Hall, N. Miyake, \& N. Enyedy (Eds.), Proceedings of CSCL '97: The Second International Conference on Computer Support for Collaborative Learning (pp. 91-100). Toronto: University of Toronto Press.

Jonassen, D. H., Peck, K. L., \& Wilson, B. G. (1999). Learning with technology: a constructivist perspective. Upper Saddle River, NJ: Prentice Hall.

Kolodner, J., \& Guzdial, M. (1996). Effects with and of CSCL: tracking learning in a new paradigm. In T. D. Koschmann (Ed.), CSCL, theory and practice of an emerging paradigm (pp. 307-320). Mahwah, NJ: Erlbaum (Lawrence).

Koschmann, T. D. (1996). Paradigm shifts and instructional technology: an introduction. In T. D. Koschmann (Ed.), Computers, cognition, and work: CSCL, theory and practice of an emerging paradigm (pp. 1-21). Mahwah, NJ: Erlbaum (Lawrence).

Larkin, J. H., \& Simon, H. A. (1995). Why a diagram is (sometimes) worth ten thousand words. In J. Glasgow, N. N. Narayanan, \& B. Chandrasekaran (Eds.), Diagrammatic reasoning; cognitive and computational perspectives (pp. 69-109). Menlo Park, CA: AAAI Press/MIT Press. (Reprinted from Cognitive Science, 1987, 11, 65-99)

Lee, J., \& Lai, K.-Y. (1991). What's in design rationale? Human-Computer Interaction, 6, 251-280.

Lesgold, A. (1998). Multiple representations and their implications for learning. In M. W. van Someren, P. Reimann, H. P. A. Boshuizen, \& T. de Jong (Eds.), Learning with multiple representations (pp. 307-319). Amsterdam: Pergamon Press.

Lesgold, A., Katz, S., Greenberg, S., Hughes, E., \& Eggan, G. (1991). Extensions of intelligent tutoring paradigms to support collaborative learning. In S. Dijkstra, H. P. M. Krammer, \& J. J. G. van Merriënboer (Eds.), Instructional models in computer-based learning environments (pp. 291-311). Berlin: Springer.

MacLean, A., Young, R., Bellotti, V., \& Moran, T. P. (1991a). Design space analysis: bridging from 
theory to practice via design rationale (EPC-1991-128). Cambridge: Rand Xerox Research Centre Cambridge Laboratory.

MacLean, A., Young, R. M., Bellotti, V. M. E., \& Moran, T. P. (1991b). Questions, options and criteria: elements of design space analysis. Human-Computer Interaction, 6, 201-250.

Paas, F. G. W. C., \& van Merriënboer, J. J. G. (1994). Instructional control of cognitive load in the training of complex cognitive tasks. Educational Psychology Review, 6, 351-371.

Paolucci, M., Suthers, D., \& Weiner, A. (1995). Belvedere: stimulating students' critical discussion. CHI95 Conference Companion, Interactive Posters, 7-11 May Denver CO (pp. 123-124).

Paolucci, M., Suthers, D., Weiner, A., \& Lesgold, A. (1996). Automated advice-giving strategies for scientific inquiry. In C. Frasson, G. Gauthier, \& A. Lesgold (Eds.), Lecture notes in computer science. Intelligent Tutoring Systems, Third International conference, ITS '96, Montreal, Canada (pp. 372381). New York: Springer.

Pea, R. D. (1993). Practices of distributed intelligence and designs for education. In G. Salomon (Ed.), Distributed cognition: psychological and educational considerations (pp. 47-87). Cambridge: Cambridge University Press.

Rittel, H. W. J. (1984). Second-generation design methods (interview by Donald P. Grant and Jean-Pierre Protzen). In N. Cross (Ed.), Developments in design methodology (pp. 317-328). Chichester: Wiley. (Reprinted from The DMG 5th anniversary Report, DMG Occasional paper No. 1, 1972, pp. 5-10.)

Rittel, H. W. J., \& Webber, M. M. (1984). Planning problems are wicked problems. In N. Cross (Ed.), Developments in design methodology (pp. 135-144). Chichester: Wiley. (Reprinted from Policy Sciences, 4, 1973, 155-169)

Scaife, M., \& Rogers, Y. (1996). External Cognition: how do graphical representations work? International journal of human-computer studies, 45, 185-213.

Scardamalia, M. A., \& Bereiter, C. (1994). Computer support for knowledge building communities. The journal of the learning sciences, 3, 265-283.

Scardamalia, M. A., Bereiter, C., Maclean, R. S., Swallow, J., \& Woodruff, E. (1989). Computer-supported intentional learning environments. Journal of Educational Computing Research, 5, 51-68.

Schijf, H. J. M., \& Simon, H. A. (1998). One person: multiple representations: an analysis of a simple, realistic multiple representation learning task. In M. W. van Someren, P. Reimann, H. P. A. Boshuizen, \& T. de Jong (Eds.), Learning with multiple representations (pp. 197-236). Amsterdam: Pergamon Press.

Smolensky, P., Fox, B., King, R., \& Lewis, C. (1988). Computer-aided reasoned discourse or, how to argue with a computer. In R. Guindon (Ed.), Cognitive science and its applications for human-computer interaction (pp. 109-162). Hillsdale, NJ: Erlbaum (Lawrence).

Stenning, K. (1998). Representation and conceptualisation in educational communication. In M. W. van Someren, P. Reimann, H. P. A. Boshuizen, \& T. de Jong (Eds.), Learning with multiple representations (pp. 320-333). Amsterdam: Pergamon Press.

Stenning, K., \& Oberlander, J. (1995). A cognitive theory of graphical and linguistic reasoning: logic and implementation. Cognitive science, 19, 97-140.

Suthers, D. (1995). Designing for internal vs external discourse in groupware for developing critical discussion skills. In CHI'95 Research Symposium, Denver.

Suthers, D. (1998a) Representations for scaffolding collaborative inquiry on ill-structured problems. http://advlearn.lrdc.pitt.edu/advlearn/papers/aera98.pdf. (Accessed: 26-11-1998.)

Suthers, D. (1998b). Technical report computer aided education and training initiative. Pittsburgh: Learning Research and Development Center, University of Pittsburgh.

Suthers, D., \& Weiner, A. (1995) Groupware for developing critical discussion skills. http://wwwcsc195.indiana.edu/csc195/suthers.html. (Accessed: 14-07-1998.)

Suthers, D. D., Toth, E. E., \& Weiner, A. (1997). An integrated approach to implementing collaborative inquiry in the classroom. In R. Hall, N. Miyake, \& N. Enyedy (Eds.), Proceedings of CSCL '97: The Second International Conference on Computer Support for Collaborative Learning (pp. 272-279). Toronto: University of Toronto Press.

Sweller, J., van Merriënboer, J. J. G., \& Paas, F. G. W. C. (1998). Cognitive architecture and instructional design. Educational Psychology Review, 10, 251-296.

Toth, J. A., Suthers, D., \& Weiner, A. (1997). Providing expert advice in the domain of collaborative 
scientific inquiry. In B. Du Boulay, \& R. Misoguchi (Eds.), Artificial intelligence in education; knowledge and media in learning systems. Proceedings of AI-ED 97 World Conference on Artificial Intelligence in Education (pp. 301-308). Amsterdam: IOS Press.

Toulmin, S. E. (1958). The uses of argument. Cambridge: Cambridge University Press.

Toulmin, S. E., Rieke, R. D., \& Janik, A. (1984). An introduction to reasoning (2nd ed.). New York: Macmillan.

Veerman, A. (2000). Computer-supported collaborative learning through argumentation. PhD dissertation, University of Utrecht

Veerman, A. L., \& Treasure-Jones, T. (1999). Software for problem solving through collaborative argumentation. In J. Andriessen, \& P. Coirier (Eds.), Foundations of argumentative text processing (pp. 203-229). Amsterdam: University of Amsterdam Press.

Zhang, J. (1997). The nature of external representations in problem solving. Cognitive Science, 21, $179-217$

Zhang, J., \& Norman, D. A. (1994). Representations in distributed cognitive tasks. Cognitive Science, $18,87-122$.

Zumbach, J., \& Reimann, P. (1999). Combining computer-supported collaborative argumentation and problem-based learning: an approach for designing online learning environments. In Computer Supported Collaborative Learning '99, Stanford, CA. 